\title{
Chilled disks in ultraluminous X-ray sources
}

\author{
Roberto Soria, ${ }^{1}$ Zdenka Kuncic, ${ }^{2}$ and Anabela C. Gonçalves ${ }^{3,4}$
}

${ }^{1}$ Harvard-Smithsonian CfA, 60 Garden st, Cambridge, MA 02138, USA

${ }^{2}$ School of Physics, University of Sydney, NSW 2006, Australia

${ }^{3}$ LUTH, Observatoire de Paris-Meudon, 5 Place Jules Janssen, 92195 Meudon, France

${ }^{4}$ CAAUL, Observatorio Astronomico de Lisboa, Tapada da Ajuda, 1349-018 Lisboa, Portugal

email: rsoria@cfa.harvard.edu,z.kuncic@physics.usyd.edu.au, anabela.darbon@obspm.fr

\begin{abstract}
If the standard disk-blackbody approximation is used to estimate black hole (BH) masses in ultraluminous X-ray sources (ULXs), the inferred masses are $\sim 1000 M_{\odot}$. However, we argue that such an approximation cannot be applied to ULXs, because their disks are only radiating a small fraction of the accretion power, and are therefore cooler than they would be in a thermal-dominant state, for a given BH mass. Instead, we suggest that a different phenomenological approximation should be used, based on three observable parameters: disk luminosity, peak temperature, and ratio between thermal and non-thermal emission. This method naturally predicts masses $\sim 50 M_{\odot}$, more consistent with other theoretical and observational constraints.
\end{abstract}

Keywords. X-rays: binaries, black hole physics, accretion, accretion disks

\section{Mass estimates from thermal disk spectra}

The masses of the accreting BHs in ULXs, and hence their nature and physical origin, are still unknown. In the absence of direct kinematic measurements, indirect methods based on X-ray spectral modelling have been used, by analogy with stellar-mass BH X-ray binaries in our Galaxy. For stellar-mass BHs in the high/soft state, most of the accretion power is radiated by the disk, and the X-ray spectrum is well fitted by a multicolour blackbody (Shakura \& Sunyaev 1973; Frank, King \& Raine 2004). The fitted peak temperature $T_{0}$ and the integrated disk-blackbody luminosity $L_{0}$ are simply related to the mass accretion rate $\dot{M}$ and the size of the inner disk $R_{\text {in }}$ (Makishima et al. 1986):

$$
\begin{aligned}
L_{0} & \approx 4 \pi \sigma T_{0}^{4} R_{\mathrm{in}}^{2} \\
L_{0} & =\eta \dot{M} c^{2} \\
\sigma T_{0}^{4} & \approx \frac{3 G M \dot{M}}{8 \pi R_{\mathrm{in}}^{3}}
\end{aligned}
$$

where we have ignored a factor related to the no-torque condition at $R_{\text {in }}$ and a hardening factor, whose combined effect is $\approx 1.35$ (Fabian, Ross \& Miller 2004). The radiative efficiency $\eta \approx 0.1-0.3$ for a source in a high/soft state.

¿From (1.1), (1.2), and (1.3), one can estimate the BH mass:

$$
M \approx \frac{c^{2} \eta L_{0}^{1 / 2} T_{0}^{-2}}{3 G(\sigma \pi)^{1 / 2}} \approx 5.6\left(\frac{\eta}{0.2}\right)\left(\frac{L_{0}}{5 \times 10^{38} \mathrm{erg} \mathrm{s}^{-1}}\right)^{1 / 2}\left(\frac{T_{0}}{1 \mathrm{keV}}\right)^{-2} M_{\odot} .
$$

Studies of Galactic stellar-mass BHs show that the spectroscopic mass estimate (1.4) is in agreement (within a factor of 2) with the kinematic mass. 


\section{Spectroscopic mass estimates for power-law-dominated ULXs}

Let us assume that the "soft-excess" component in ULXs is indeed thermal emission from a disk (see Gonçalves \& Soria 2006 for an alternative scenario). Direct application to (1.4) of the observed values of their X-ray luminosity $\left(L_{0} \sim 10^{40} \mathrm{erg} \mathrm{s}^{-1}\right)$ and colour temperature $\left(k T_{0} \approx 0.15 \mathrm{keV}\right)$ has led to the suggestion that ULXs may contain intermediate-mass BHs with masses $\sim 1000 M_{\odot}$ (Miller, Fabian \& Miller 2004).

However, the thermal component is only a small fraction $(\sim 10 \%)$ of the X-ray emission in ULX spectra. In the inner region, at $R_{\text {in }} \leqslant R \leqslant R_{\mathrm{c}}$, most of the accretion power is released via non-thermal processes. This implies that the inner disk is cooler than a standard disk, because it radiates only a flux

$$
\sigma T(R)^{4} \approx \frac{3 G M \dot{M}}{8 \pi R^{3}}-F_{\mathrm{nr}}(R)<\frac{3 G M \dot{M}}{8 \pi R^{3}}
$$

where $F_{\mathrm{nr}}(R)$ is the energy flux released via non-radiative processes, for example transferred to a corona or outflow via magnetic stresses (Kuncic \& Bicknell 2004). If $T(R)$ increases more slowly that $R^{-1 / 2}$ for $R \rightarrow R_{\text {in }}$, the maximum contribution to the disk emission occurs at $R \approx R_{\mathrm{c}}, T \approx T\left(R_{\mathrm{c}}\right)$ (peak in the fitted spectrum). For simplicity, here we assume that $T=T\left(R_{\mathrm{c}}\right)=$ constant inside $R_{\mathrm{c}}$; only a fraction $\beta<1$ of the disk emission from the inner region is directly visible, depending on the optical depth of the scattering region. We can now re-write (1.1), (1.2), (1.3) as:

$$
\begin{aligned}
L_{0} & =4 \pi R_{\mathrm{c}}^{2} \sigma T_{0}^{4}+2 \pi\left(R_{\mathrm{c}}^{2}-R_{\mathrm{in}}^{2}\right) \sigma T_{0}^{4} \times \beta \approx 4 \pi R_{\mathrm{c}}^{2} \sigma T_{0}^{4} \times(1+\beta / 2) \\
L_{0} & =f \eta \dot{M} c^{2} \\
\sigma T_{0}^{4} & \equiv \sigma T\left(R_{\mathrm{c}}\right)^{4} \approx \frac{3 G M \dot{M}}{8 \pi R_{\mathrm{c}}^{3}} .
\end{aligned}
$$

where $L_{0}$ is the total radiative luminosity of the disk, $T_{0} \equiv T\left(R_{\mathrm{C}}\right)$ is the peak temperature, and $f \sim 0.1$ is the fraction of accretion power radiated by the disk; $f$ cannot be directly measured, but we can estimate it based on the fitted ratio of soft thermal emission over total X-ray luminosity. We can then solve (2.1), (2.2), (2.3) for $M, \dot{M}$ and $R_{\mathrm{c}}$ as a function of the observable quantities $f, T_{0}$ and $L_{0}$. In particular, for the $\mathrm{BH}$ mass we obtain:

$$
M \approx \frac{49.8}{(1+\beta / 2)^{3 / 2}}\left(\frac{\eta}{0.2}\right)\left(\frac{f}{0.1}\right)\left(\frac{L_{0}}{2 \times 10^{39} \mathrm{erg} \mathrm{s}^{-1}}\right)^{1 / 2}\left(\frac{T_{0}}{0.15 \mathrm{keV}}\right)^{-2} M_{\odot}
$$

We conclude that the fitted spectral features of ULXs (X-ray luminosity, temperature and ratio of thermal/non-thermal contribution) suggest masses $\sim 50 M_{\odot}$. This is at the extreme end of, but still consistent with models of stellar evolution. If that is the case, the emitted luminosity is a few times the Eddington luminosity $L_{\text {Edd }}$, but the disk radiative contribution alone is $\lesssim L_{\text {Edd }}$. The rest is generated outside the disk by non-thermal processes, which dominate at radii $\lesssim R_{\mathrm{c}} \sim 100$ gravitational radii.

\section{References}

Fabian, A. C., Ross, R. R. \& Miller, J. M. 2004, MNRAS, 355, 359

Frank, J., King, A. \& Raine, D. 2002, Accretion Power in Astrophysics (Cambridge University Press)

Gonçalves, A. C. \& Soria, R. 2006, MNRAS, 371, 673

Kuncic, Z. \& Bicknell, G. V. 2004, AJ, 616, 669

Makishima, K. et al. 1986, AJ, 308, 635

Miller, J. M., Fabian, A. C. \& Miller, M. C. 2004, ApJ, 614, L117

Shakura, N. I. \& Sunyaev, R. A. 1973, A\&A, 24, 337. 